\title{
La invención del neutrino: un análisis epistemológico
}

\author{
Alejandro Cassini
}

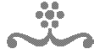

RESUMEN

Hacia 1930, el problema de explicar la desintegración radiactiva Beta produjo un estado de crisis en el dominio de la física de partículas. La conservación de la energía, del momento y de la estadística cuántica parecían amenazadas. Pauli consiguió resolver todas esas anomalías postulando la existencia de neutrinos dentro del núcleo atómico. Pero lo hizo al costo de presuponer un modelo del núcleo atómico que resultó insostenible después del descubrimiento del neutrón en 1932. En 1933, Fermi concibió la explicación de la desintegración Beta que todavía consideramos correcta. En este artículo, hago una revisión de las virtudes explicativas de la hipótesis del neutrino. Argumento que la invención de Pauli era ad hoc en 1930 pero se volvió contrastable en el contexto de la teoría de Fermi. Concluyo, entonces, afirmando que no había evidencia suficiente como para aceptar la existencia del neutrino antes del segundo experimento de Cowan y Reines de 1956.
\end{abstract}

Palabras-cLave • Desintegración Beta. Neutrino. Leyes de conservación. Estadística cuántica. Descubrimiento científico.

\section{INTRODUGGIÓN}

La mayor parte de las numerosas partículas elementales y subatómicas actualmente conocidas se descubrieron sin que ninguna teoría predijera su existencia antes del descubrimiento (cf. Cassini, 2001). El neutrino, en cambio, pertenece al reducido grupo de las que se postularon de manera puramente teórica para explicar ciertos fenómenos aparentemente anómalos y, algún tiempo después, se descubrieron de manera experimental. El caso del neutrino resulta peculiar por el hecho de que la primera observación de esta clase de partícula, o, al menos, la primera evidencia observacional de su existencia, se obtuvo más de dos décadas después de su postulación por razones teóricas. En el tiempo intermedio, la hipótesis de la existencia del neutrino se incorporó a una teoría general de la desintegración radiactiva, teoría que se utilizó de manera muy exitosa para explicar diversos procesos subatómicos. Por esta razón, la comunidad de los físicos aceptó la existencia del neutrino mucho antes de que se hubiera obtenido 
por medios experimentales suficiente evidencia de su existencia. Este es, sin duda, un hecho atípico. Los científicos generalmente no admiten nuevos tipos de entidades sin disponer de evidencia observacional de que tales entidades son efectivamente reales y no meros constructos teóricos o ficciones explicativas útiles. En esta ocasión, sin embargo, no procedieron de esa manera. Se trata de una circunstancia lo suficientemente intrigante como para merecer un análisis epistemológico atento. Eso es lo que intentaré hacer en este trabajo.

La compleja historia de la hipótesis del neutrino, desde su formulación teórica hasta su confirmación experimental, ha sido relatada con frecuencia y no es mi objetivo contribuir aquí a completar esa historia. La obra más importante sobre el tema es la de Allan Franklin (2004), que supera a todas las anteriores ofreciendo un análisis detallado y erudito de este proceso (cf. Allen, 1958; Solomey, 1997). No obstante, los comentarios epistemológicos de Franklin, más bien breves y dispersos, son, en mi opinión, a menudo insuficientes y a veces incluso poco acertados. Hasta donde llega mi conocimiento, todavía no se ha hecho un estudio epistemológico adecuado del descubrimiento del neutrino y de sus implicaciones para la filosofía de la ciencia. El presente trabajo constituye un primer paso hacia ese objetivo.

Para los fines del análisis, dividiré el proceso de descubrimiento del neutrino en tres etapas. La primera, que llamo etapa de la invención, transcurrió entre 1930 y 1931 cuando Wolfgang Pauli propuso la hipótesis del neutrino para explicar el fenómeno aparentemente anómalo de la desintegración radiactiva de ciertos átomos, fenómeno conocido desde hacía tiempo como desintegración $\beta$. La segunda, la etapa de la explicación, comenzó en 1933 cuando Enrico Fermi incorporó la hipótesis del neutrino a su teoría de la interacción débil y explicó satisfactoriamente diversos aspectos del fenómeno de la desintegración $\beta$. La tercera, la etapa del descubrimiento, comenzó en $195^{3}$ cuando Clyde Cowan y Frederik Reines realizaron su primer experimento para detectar neutrinos. Esta última etapa se cerró en 1960 cuando se publicaron los datos definitivos que permitieron afirmar, no sin reservas, que efectivamente se habían observado neutrinos.

En este trabajo estudiaré las etapas de la invención y explicación del neutrino. ${ }^{\mathbf{1}}$ En la sección 2, presentaré, de manera necesariamente esquemática, los problemas que planteaba la desintegración radiactiva $\beta$ antes de 1930. En la sección 3, examinaré la manera en que Pauli introdujo la hipótesis del neutrino y las razones que adujo para justificar su hipótesis. En la sección 4, indicaré cómo la teoría de la desintegración $\beta$ de Fermi incorporó la hipótesis del neutrino. En esas tres secciones, citaré in extenso

1 La etapa del descubrimiento, que plantea problemas específicos para la filosofía de la experimentación, espero abordarla en un trabajo posterior. 
varios pasajes de las fuentes correspondientes. ${ }^{2}$ En la sección 5 , haré un análisis epistemológico de las dos primeras etapas del proceso que condujo al descubrimiento del neutrino. Allí intentaré responder a las siguientes preguntas. Primera, ¿qué tipo de argumentos se ofrecieron para justificar la invención de la hipótesis del neutrino? Segunda, ¿cómo debe evaluarse esa hipótesis desde el punto de vista epistemológico? Tercera, ¿era razonable aceptar la existencia del neutrino antes de que se lo detectara experimentalmente? Estas preguntas, no es necesario decirlo, son sólo algunas de las muchas cuestiones filosóficas que podrían plantearse en torno al proceso de descubrimiento del neutrino. No obstante, creo que las tres que he formulado tocan los problemas epistemológicos más importantes.

\section{Las anomalías de LA DESintegración $\beta$}

Desde el momento en que Henri Becquerel descubrió en 1896 los "rayos uránicos", la radiactividad no dejó de plantear a los físicos un enigma tras otro (cf. Pais, 1988, cap. 2, 3; Jensen, 2000; Kragh, 1997). Así, a comienzos del siglo xx, los físicos tenían ante sí tres tipos diferentes de radiación, llamadas convencionalmente $\alpha, \beta$ y $\gamma$, cuyas propiedades podían estudiarse experimentalmente pero cuyo origen carecía de toda explicación teórica.

La desintegración $\beta$ se concebía como el proceso por el cual determinados elementos que poseen radiactividad natural se transforman en otros elementos produciendo, a la vez, radiación bajo la forma de un flujo de electrones de alta energía. Así, por ejemplo, el carbono radiactivo $\left({ }_{6} \mathrm{C}^{14}\right)$ origina nitrógeno $\left({ }_{7} \mathrm{~N}^{14}\right)$. La reacción, tal como se la podía observar experimentalmente a comienzos del siglo $\mathrm{xx}$, era la siguiente: ${ }_{6} \mathrm{C}^{14}$ $\rightarrow{ }_{7} \mathrm{~N}^{14}+e$. Los elementos que experimentan desintegración $\beta$ “decaen" en otro elemento, que tiene un número atómico inmediatamente superior y el mismo número másico, además, de emitir radiación $\beta$ (en símbolos: $\mathrm{Z}_{\mathrm{Z}}^{\mathrm{A}} \rightarrow_{\mathrm{Z}_{+}} \mathrm{Y}^{\mathrm{A}}+\mathrm{e}^{-}$, donde $\mathrm{X}$ e $\mathrm{Y}$ son dos elementos diferentes, $z$ es la carga eléctrica del núcleo - el número atómico - y $a$ es el número másico del núcleo). Puede verse que la carga eléctrica se conserva en el proceso de desintegración, ya que, si bien el núcleo atómico gana una carga positiva, también emite un electrón, que tiene carga negativa.

2 Un relato minucioso y sumamente erudito de la física de partículas antes y después de la hipótesis del neutrino, junto con un catálogo exhaustivo de las fuentes, se encuentra en la obra de Abraham Pais (1988). El libro de Jensen (2000) es un estudio detallado de la historia de la desintegración $\beta$. La compilación de Winter (2000) contiene trabajos originales sobre la física del neutrino. No es posible citar aquí la totalidad de las fuentes relevantes para el descubrimiento del neutrino. Para ello, remito al libro de Franklin (2004) que debe completarse con las obras de Pais (1988) y de Enz (2002) para algunas omisiones importantes. 
Sin embargo, otras leyes clásicas de conservación parecían estar amenazadas. Esta reacción presentaba tres problemas que eran muy difíciles de explicar: en primer lugar, la radiación $\beta$ tenía un espectro continuo, lo cual parecía violar la ley de conservación de la energía; en segundo lugar, en la reacción parecía violarse una ley de conservación de la estadística cuántica de los núcleos atómicos; en tercer lugar, en el curso de la reacción, tampoco parecía conservarse el spin, lo cual implicaba una violación de la ley de conservación del momento angular. Consideremos estas tres anomalías según el orden cronológico en que fueron descubiertas.

La naturaleza de la radiación $\beta$ se develó rápidamente, pero el mecanismo por el cual se originaba permaneció oculto por mucho tiempo. En el año 1900, Pierre y Marie Curie midieron la carga eléctrica de los rayos $\beta$ y encontraron que era negativa. Dos años después, Walter Kaufmann finalizó una serie de experimentos destinados a medir la masa de los rayos. Concluyó que esa masa era idéntica, dentro del margen de error observacional, a la de los rayos catódicos. Desde ese momento, la comunidad de los físicos aceptó que los rayos $\beta$ eran electrones emitidos por las sustancias radiactivas. Por su parte, en 1913, Niels Bohr argumentó que dichos electrones debían provenir del núcleo atómico y no de las órbitas exteriores del átomo (cf. Bohr, 1913).

En 1914, se descubrió el carácter continuo del espectro de la radiación $\beta$. Este descubrimiento fue la culminación de un largo y complicado proceso de investigación experimental en el que intervinieron numerosos físicos de diferentes países (cf. Franklin, 2002, cap. 8; 2004, p. 28-60; Jensen, 2000). James Chadwick (1914) fue quien finalmente estableció la continuidad de ese espectro. Esa era una anomalía de grandes proporciones, por lo que es importante detenerse a aclarar en qué consiste el carácter anómalo de dicho espectro.

¿Qué significa que el espectro de la radiación $\beta$ sea continuo? Esencialmente, que los electrones emitidos por una sustancia radiactiva tienen energías que toman cualquier valor entre cero y un máximo, Emáx., que es característico de cada sustancia. Las mediciones realizadas en 1914, sobre diferentes sustancias radiactivas mostraban que Emáx. era siempre igual a la diferencia de niveles energéticos de los átomos que emiten radiación (es decir, Emáx. $=$ En “Em). En consecuencia, el espectro de la radiación debía ser discreto (como ya se conocía que lo eran los espectros de la radiación $\alpha$ y $\gamma$ ). Por ello, el carácter continuo de la radiación $\beta$ resultaba inexplicable, porque si los átomos tenían una energía cuantificada que sólo tomaba valores discretos y bien determinados, como había postulado Bohr (1913), necesariamente debían emitir radiación de espectros discretos. El problema consistía, entonces, en explicar por qué en la radiación $\beta$ se emitían electrones que tenían energías menores que Emáx. y que, además, formaban un espectro continuo. Todos los electrones emitidos que tuvieran una energía menor que Emáx. habían perdido energía de alguna manera. Si no quería 
ponerse en cuestión la ley de conservación de la energía, era necesario encontrar algún mecanismo causal que explicara dicha pérdida.

Durante las dos décadas posteriores al descubrimiento de Chadwick se propusieron muy diversas hipótesis para explicar el espectro continuo de la radiación Beta, pero ninguna resultó exitosa (cf. Pais, 1988; Jensen, 2000; Franklin, 2004). Una clase general de hipótesis era la llamada teoría del proceso secundario, que consistía en suponer que la desintegración $\beta$ no era un proceso primario, es decir, originado en los núcleos atómicos, sino el resultado de la interacción de los rayos $\beta$ con el entorno. Una de las hipótesis de esa clase sostenía que todos los electrones salen del núcleo con una energía igual a Emáx., pero pierden parte de esa energía al chocar con otros átomos de la sustancia radiactiva. Si esto es así, se sigue que una muestra de cualquier sustancia radiactiva que emita rayos $\beta$ se debe calentar espontáneamente, puesto que la energía liberada en los choques se disipa bajo la forma de calor. En 1927, Charles Ellis y William Wooster realizaron los experimentos más precisos de su época para detectar el supuesto calentamiento, con resultado negativo. Concluyeron que la desintegración $\beta$ era un proceso primario y que los electrones emergían del núcleo con una distribución continua de energía (cf. Ellis \& Wooster, 1927). Después de 1927, la comunidad científica desestimó la hipótesis de la pérdida de energía por procesos secundarios considerándola refutada. Debe advertirse, sin embargo, que la predicción del calentamiento no se deduce sólo de la hipótesis de los choques, sino que, además, requiere como premisa la ley de conservación de la energía. Aquí tenemos un buen ejemplo de holismo epistemológico, que nos impide saber cuál de las dos hipótesis ha sido refutada. El resultado experimental de la ausencia de calentamiento también podría haberse interpretado como una refutación de la ley de conservación de la energía, aunque pocos físicos estaban dispuestos a hacerlo.

En 1929, Bohr revivió nuevamente la idea de que la ley de conservación de la energía se viola en los procesos de desintegración radiactiva. ${ }^{3}$ Sostuvo que esa ley se cumple solo estadísticamente, para grandes cantidades de átomos, pero no en cada proceso atómico individual. La mecánica cuántica, sin embargo, había llegado a una formulación coherente suponiendo la estricta conservación de la energía en todo proceso microscópico. Bohr era consciente de esa situación y respondió a sus críticos afirmando que la nueva mecánica cuántica había alcanzado ya los límites de su desarrollo y que, en consecuencia, eran necesarias nuevas leyes para explicar procesos como la desintegración $\beta$ (cf. Bohr, 1986 [1929]). La hipótesis de Bohr recibió una fría adhesión

3 Bohr ya había elaborado una teoría donde la energía no se conservaba en los procesos subatómicos individuales, la efímera teoría conocida como BKS (cf. Peierls, 1986a; Bohr; Kramers \& Slater, 1924). En ese momento, Bohr no publicó sus ideas, resumidas en un manuscrito inédito (cf. Bohr, 1986 [1929]). 
por parte de los físicos. La mayoría pensaba como Paul Dirac, cuando en una carta a Bohr, de noviembre de 1929, le expresó que "preferiría mantener la rigurosa conservación de la energía a cualquier costo" (Bohr apud Pais, 1988, p. 313).

La posición de Bohr podía sustentarse en el hecho de que en ese momento había muy poca evidencia empírica acerca de la conservación de la energía en eventos microscópicos. La mayor parte de la evidencia provenía de la física clásica y de la observación de fenómenos macroscópicos. Así pues, argumentaba Bohr, como la mecánica cuántica nos ha forzado a renunciar a diversas leyes clásicas (entre las que incluía el principio de causalidad), también puede llevarnos a abandonar la ley de conservación de la energía. En el caso de la desintegración $\beta$, Bohr no veía ninguna evidencia empírica o teórica para mantener esa ley.

El descubrimiento del efecto Compton, la dispersión de la luz por los electrones en reposo, había mostrado, sin embargo, la aplicabilidad de la ley de conservación de la energía a los procesos subatómicos (cf. Compton, 1923). El efecto Compton se considera habitualmente como una prueba de la naturaleza corpuscular de la luz y, a la vez, como una confirmación de que la energía y el momento se conservan en los procesos individuales de dispersión de partículas (cf. Shamos, 1987 [1959], p. 329; Bohm, 1989 [1951], p. 33). ${ }^{4}$ Sin embargo, no puede decirse que el efecto Compton representa una confirmación de esas leyes por sí mismas. Compton no dedujo ninguna consecuencia observacional de las leyes de conservación tomadas aisladamente, sino de un conjunto muy amplio de hipótesis. La ley de conservación de la energía tiene un carácter puramente teórico y no predice por sí sola ningún evento observable. Compton utilizó diversas teorías presupuestas, además de las leyes de conservación, para obtener sus ecuaciones, entre otras, la teoría de la relatividad especial y la teoría cuántica de la luz de Einstein. Supuso que la radiación incidente estaba formada por cuantos de luz de energía $E=h v$ y momento lineal $p=h v / c$, y que ambas cantidades se conservaban después de la interacción entre los cuantos de luz y los electrones, a la que concibió como un caso de colisión de partículas. Los fenómenos de dispersión que observó Compton (la disminución de la frecuencia de los rayos X dispersados por átomos de grafito) confirmaban globalmente al sistema total de hipótesis del cual se deducían sus ecuaciones. Las leyes de conservación no recibían una confirmación independiente. No obstante, es indudablemente cierto que el efecto Compton probó, en el contexto de todo un sistema teórico, la aplicabilidad de las leyes de conservación del momento y la energía a los procesos individuales de dispersión entre partículas elementales.

4 Generalmente, se considera también que sólo después de conocidos los resultados de Compton se aceptó sin reservas la hipótesis del cuanto de luz de Einstein. Sobre todo este proceso véase la obra exhaustiva de Stuewer (1975). La evidencia a favor y en contra del cuanto de luz en la época del experimento de Compton ha sido reevaluada por Brush (2007). 
Con todo, la desintegración $\beta$ es un proceso físico de una especie completamente diferente del efecto Compton, ya que no involucra la interacción entre los electrones de los átomos y los cuantos de luz incidentes del exterior. Por esa razón, dicho efecto no constituía evidencia de la validez de las leyes de conservación en el campo de los fenómenos radiactivos. Era perfectamente concebible que la desintegración $\beta$ y el efecto Compton estuvieran gobernados por leyes totalmente diferentes.

La segunda anomalía de la desintegración $\beta$ era que ésta parecía violar las leyes de la estadística cuántica. La aclaración de esa anomalía requiere un breve análisis del modelo del núcleo atómico vigente hacia 1930. En ese momento, se consideraba que el núcleo estaba compuesto por protones y electrones, de los cuáles se conocían la masa, la carga eléctrica y el spin. Protones y electrones eran, pues, partículas de spin semientero $\left( \pm^{1 / 2} h / 2 \pi\right)$ que obedecían al principio de exclusión de Pauli y estaban sometidas a la estadística de Fermi-Dirac. El número total de fermiones del núcleo $N f$ se suponía igual a $N p+N e$, esto es, a la suma del número de protones y electrones. $\mathrm{Si} A$ es el número másico del núcleo y $Z$ su carga eléctrica, $N f$ debía ser igual a $2 A-Z$. Se admitía que $A$ era igual a $N p$ y $Z$ era igual a $N p-N e$. El átomo se completaba con un número igual a $Z$ electrones orbitando alrededor del núcleo. La masa y la carga del núcleo eran observables, esto es, determinables por medio de alguna medición, por indirecta que fuera. El número y la clase de partículas que componían el núcleo, en cambio, no eran observables y debían conjeturarse. Dados los recursos conceptuales y los datos disponibles en ese momento, el llamado modelo protón-electrón parecía ser la única explicación razonable de la estructura del núcleo.

De acuerdo con ese modelo, un proceso de desintegración radiactiva $\beta$ como el que experimentaba el ${ }_{6} \mathrm{C}^{14}$ debía explicarse de la siguiente manera. El núcleo del ${ }_{6} \mathrm{C}^{14}$, que contiene 22 fermiones ( 14 protones y 8 electrones), emite un electrón y se convierte en un núcleo de ${ }_{7}{ }^{14}$, que contiene 21 fermiones (14 protones y 7 electrones). El electrón emitido por el núcleo de carbono se observa, a su vez, como radiación $\beta$. Como consecuencia de ello, el spin de los respectivos núcleos debía cambiar de entero a semientero. De acuerdo con la regla formulada por Eugene Wigner en 1928, el spin de una partícula compuesta de $N$ fermiones es semientero si $N$ es impar, y es entero si $N$ es par. En el primer caso, la partícula compuesta obedece a la estadística de Fermi-Dirac y en el segundo a la de Bose-Einstein. Pauli llamó "ley de la alternancia" (Wechselsatz) a esta regularidad, a la que, pese a que todavía no se había demostrado en general la conexión entre el spin y la estadística de una partícula, consideraba bien establecida, tanto como a las propias leyes de conservación. ${ }^{5}$ Según la ley de la altyernancia, el ${ }_{6} \mathrm{C}^{14}$ debía tener spin entero, pero el ${ }_{7} \mathrm{~N}^{14}$, que resultaba de la desintegración $\beta$ de éste debía

5 La prueba general de la relación entre spin y estadística cuántica la dio el propio Pauli (1940). 
tener spin semientero. Tal predicción, sin embargo, parecía refutada por los experimentos de Walter Heitler y Gerhard Herzberg de 1929, cuyas mediciones de las líneas espectrales del nitrógeno indicaban que éste tenía spin 1 y, por tanto, obedecía la estadística de Bose-Einstein. A la luz de ete resultado, Heitler y Herzberg habían concluido que la regla de Wigner no tenía validez para los núcleos atómicos (cf. Heitler \& Herzberg, 1929). Una dificultad análoga se descubrió simultáneamente respecto del isótopo radiactivo del litio ${ }_{3} \mathrm{Li}^{6}$. El problema llegó a ser conocido como el de la "estadística errónea" y, para Pauli, representaba una anomalía fundamental de la desintegración $\beta$.

La tercera anomalía era la aparente violación de la ley de conservación del spin o momento angular intrínseco de las partículas. Si el núcleo del átomo de nitrógeno tenía efectivamente spin entero, como mostraban las observaciones, y no semientero, como predecía la ley de la alternancia, entonces, la desintegración $\beta$ del carbono implicaba una violación de la conservación del momento angular, puesto que los productos de la desintegración del núcleo de carbono, de spin entero, incluían un electrón. En el proceso, parecía haber, entonces, una pérdida o ganancia neta de momento angular intrínseco igual a $1 / 2 h / 2 \pi$.

\section{LA INVENGIÓN DEL NEUTRINO}

Hacia 1930 el problema de explicar el espectro continuo de la radiación $\beta$ estaba aún abierto, ya que se habían eliminado casi todas las explicaciones propuestas. Según relata Pauli, ${ }^{6}$ en aquel momento sólo quedaban en pie dos hipótesis teóricas rivales, ambas compatibles con los resultados experimentales. Pauli las formula de la siguiente manera:

(1) Que en aquellas interacciones responsables de la radiactividad Beta la conservación de la energía se verificara sólo estadísticamente.

(2) Que la ley de la [conservación] de la energía fuera estrictamente válida para cada proceso individual primario, pero que, junto con los electrones, fuera emitida en el curso del mismo otra radiación muy penetrante constituida por nuevas partículas neutras (Pauli, 1996 [1961], p. 247).

La primera hipótesis describe la posición de Bohr, que ya mencionamos antes. Bohr concibió sus ideas en 1929, pero sólo las publicó en 1932 (Bohr, 1986 [1932]).

6 Pauli (1961) es la fuente de todos los relatos sobre la invención del neutrino. Es el texto de una conferencia pronunciada el 21 de enero de 1957, luego corregida en 1958, y publicada póstumamente en 1961 (cf. Enz, 2002, p. 209-39). 
Entretanto, Pauli había formulado la hipótesis del neutrino, la segunda hipótesis en disputa. Pauli comunicó su invención el 14 de noviembre de 1930 en una célebre carta dirigida a los participantes de un congreso sobre el problema de la radiactividad. La parte esencial de la carta decía lo siguiente:
(...) he encontrado un remedio desesperado para preservar la "ley de la alternan- cia" de la estadística y la ley de la energía en relación con la estadística "errónea" de los núcleos de N-14 y de Li-6, así como del espectro $\beta$ continuo. Estriba en la posibilidad de que en los núcleos puedan existir partículas eléctricamente neu- tras, que denominaré neutrones, con spin $1 / 2$, que obedezcan al principio de ex- clusión y que además se diferencien de los cuantos luminosos por no viajar a la velocidad de la luz. La masa de los neutrones tendría que ser del mismo orden de magnitud que la masa del electrón y, en cualquier caso, no mayor que 0,01 veces la masa del protón. El espectro $\beta$ continuo sería entonces comprensible bajo la hipótesis de que en la desintegración $\beta$, junto con el electrón, se emite también un neutrón, de tal forma que la suma de las energías de ambos sea constante (Pauli, 1996 [1961], p. 249).

Pauli llamó “neutrón” a su hipotética partícula. En el pasaje citado, se puede advertir claramente que Pauli pensaba que tanto el neutrino como el electrón emitidos en la desintegración $\beta$ preexistían en los núcleos atómicos.

La hipótesis del neutrino de Pauli resolvía a la vez todas las anomalías de la radiación $\beta$. El neutrino debía ser el portador de la energía faltante en los productos de la desintegración y, además, dado que tenía spin $1 / 2$, restablecía el balance del momento angular y permitía conservar la estadística cuántica. Los neutrinos también debían poseer un espectro continuo de energía, pero complementario al de los electrones. La suma de las energías cinéticas de ambas partículas resultaba, entonces, constante e igual a $\mathrm{E}_{\text {máx. }}$. Con ello, quedaba explicado el espectro continuo de la radiación $\beta$.

La hipótesis del neutrino de Pauli implicaba una modificación del modelo vigente del núcleo. Si se admite que tanto los electrones como los neutrinos existen en el núcleo, es razonable suponer que se encuentran apareados. El número total de fermiones del núcleo $N f$ resulta entonces igual a $N p+N e+N v$ esto es, a la suma del número de protones, electrones y neutrinos, número que resulta igual a $3 A-2 Z$. Por otra parte, en este modelo se mantiene el supuesto de que $A$ es igual a $N p$ y $Z$ es igual a $N p-N e$. Si se supone que el neutrino tiene spin $1 / 2$ y preexiste en el núcleo, entonces, el spin del núcleo del ${ }_{7} \mathrm{~N}^{14}$ resulta entero, en concordancia con las observaciones, y no hay cambio en la estadística cuántica. 
¿Cuáles fueron las razones de Pauli para postular la existencia del neutrino? La información más explícita que ofrece Pauli se encuentra en su comunicación a la Séptima Conferencia Solvay de octubre de 1933. Después de reseñar las anomalías que presenta la desintegración $\beta$, dice lo siguiente:

(...) se presentan dos interpretaciones de las experiencias. La que defiende Bohr admite que las leyes de la conservación de la energía y del impulso no se verifican de forma total cuando se trata de un proceso nuclear en el que las partículas ligeras desempeñan un papel esencial. Esta hipótesis no me parece satisfactoria ni incluso plausible. En primer lugar, la carga eléctrica se conserva en el proceso, y no veo por qué la conservación de la carga habría de ser más fundamental que la de la energía y la del impulso. Además, son precisamente las relaciones energéticas las que regulan varias características de los espectros $\beta$ (...). Si las leyes de conservación no fueran válidas, sería preciso concluir de estas relaciones que una desintegración $\beta$ es siempre acompañada de una pérdida de energía y nunca de una ganancia; esa conclusión implica una irreversibilidad de los procesos respecto del tiempo, que no me parece aceptable (Pauli, 1996 [1961], p. 254).

Esa explicación deja bien en claro que la retención del principio de conservación de la energía no fue el único motivo que tuvo Pauli para proponer su hipótesis, como se afirma a menudo. La conservación de la estadística cuántica le parecía de fundamental importancia. En una carta a Heisenberg del 14 de julio de 1933 le dijo incluso que consideraba a la conservación de la estadística cuántica "casi más importante que las leyes de conservación de la energía y el momento" (Pauli apud Pais, 1988, p. 318; Enz, 2000, p. 228).

¿Qué valor tienen esos argumentos desde un punto de vista lógico? Ante todo, la apelación a la conservación de la carga eléctrica no tiene valor probatorio porque es perfectamente concebible que la desintegración $\beta$ satisfaga sólo una de las dos leyes. El hecho de que ambas leyes se hayan cumplido en todos los procesos físicos conocidos hasta el momento sólo brinda apoyo inductivo a la hipótesis de que también se cumplirán en la desintegración $\beta$. Es, sin duda, un apoyo inductivo fuerte, dada la enorme cantidad de fenómenos observados en los que ambas leyes se cumplen; pero, como todo argumento inductivo, no es lógicamente concluyente.

El segundo argumento apela a las consecuencias inaceptables que tendría la violación de la ley de conservación de la energía. En concreto, ella implicaría la violación de otra ley fundamental de la física, la de la reversibilidad temporal de los procesos físicos. Esta es también una ley muy general que vale en la mecánica newtoniana, en la física estadística y también en la mecánica relativista y cuántica. Se la puede enunciar 
de esta manera: sea $A, B, C$ una secuencia de eventos, de un mismo proceso físico, que ocurren en ese orden temporal; si esa secuencia es físicamente posible (esto es, compatible con las leyes aceptadas), entonces, también es físicamente posible una secuencia en la que esos eventos ocurren en el orden $C, B$, $A$. En el caso de la desintegración $\beta$, la violación se produce porque los electrones emitidos nunca ganan energía. Nuevamente, el argumento no es concluyente. Depende de cuánto se valore la retención del principio de reversibilidad temporal de los procesos físicos. Desde un punto de vista lógico, no hay ninguna imposibilidad en renunciar a cualquiera de las leyes fundamentales de la física. Dado que se trata de leyes independientes, siempre es posible rechazar una de ellas y retener a las restantes.

Sin embargo, cada ley que se elimina de una teoría tiene un costo en términos de revisión de esa teoría y de todas las que la presuponen. Cuando se suprime una ley fundamental, o una muy general y de gran alcance, el costo es muy alto porque puede ocurrir que sea necesario revisar todas las teorías que emplean dicha ley. No obstante, una revisión drástica de esta clase no siempre es necesaria. También es posible restringir el dominio de aplicación de una ley. En el caso de la conservación de la energía, podría limitarse el alcance de dicha ley al dominio macroscópico, considerando que sólo se viola en los procesos subatómicos individuales. La conservación de la energía en el nivel macroscópico sería, como Bohr había propuesto, el resultado del carácter estadístico de esa ley en el nivel microscópico, donde se cumple sólo para agregados de grandes números de partículas, pero no para cada partícula tomada individualmente.

Esa solución, aunque plausible en general, tenía un alto costo hacia fines de la década de 1920. Después de más de veinte años de arduos esfuerzos colectivos, se había logrado construir una física cuántica coherente que parecía explicar de manera satisfactoria casi todos los fenómenos conocidos del dominio microscópico. La mecánica cuántica se había terminado de formular entre 1926 y 1927, presuponiendo la estricta conservación de la energía en todos los procesos microscópicos individuales. En el caso de la desintegración $\beta$, sin embargo, la energía no podría conservarse ni siquiera estadísticamente, ya que siempre los electrones mostraban una pérdida de energía y no parecía existir ningún proceso subatómico que compensara esa pérdida. Por otra parte, como ya hemos visto, tampoco el momento angular y la estadística cuántica parecían conservarse. Todo ello significaba una amenaza de revisiones sustanciales a la recién constituida mecánica cuántica. Además, la pérdida de las leyes de conservación habría dejado a la física sin una de sus principales herramientas heurísticas. Bohr parecía dispuesto a pagar ese costo, ${ }^{7}$ pero Pauli (y con él la mayoría de los físicos de la

7 En su manuscrito de 1929, Bohr advertía que una de las consecuencias "inquietantes" del abandono de las leyes de conservación era la pérdida de la guía que había ofrecido en el desarrollo de la teoría atómica (Bohr, 1986 [1929], p. 89). 
época) no lo estaba. La postulación del neutrino puede verse, entonces, como el resultado de un cálculo costo-beneficio acerca de cuál es la hipótesis que resuelve el mayor número de anomalías conocidas de la desintegración $\beta$ y, a la vez, implica la menor revisión de las teorías físicas aceptadas en ese momento. Desde esta perspectiva, la hipótesis de Pauli resultaba muy superior a la de Bohr: no sólo tenía menores costos, sino también mayores beneficios.

Los costos de ambas hipótesis rivales eran obviamente disímiles. La hipótesis de Pauli no implicaba la revisión de ninguna hipótesis fundamental de la mecánica cuántica o clásica, ya que era compatible con ambas. La explicación de la desintegración $\beta$ mediante la hipótesis del neutrino solamente requería expandir la teoría de las partículas subatómicas, que, por esa época, era incipiente y claramente incompleta. Por ejemplo, no era todavía capaz de explicar la constitución y la estabilidad de los núcleos atómicos. ${ }^{8}$ Además, también exigía modificar el modelo vigente del núcleo atómico, que en realidad era muy mal conocido y tenía serias dificultades teóricas.

Hacia el final de su vida, Pauli se refirió a la hipótesis del neutrino como "esa loca criatura de mi crisis vital" (apud Pais, 1988, p. 314). Sin embargo, si se la considera relativamente a la hipótesis rival de Bohr, la estrategia de Pauli era la más conservadora. Por otra parte, la postulación de entidades inobservadas, pero observables en principio, para explicar fenómenos aparentemente anómalos, que hoy nos parece casi rutinaria, ya tenía antecedentes en la física, tanto exitosos como fallidos. Resulta difícil, entonces, comprender las razones de la resistencia de Pauli y de otros físicos (como Bohr y Dirac) a la simple estrategia de postular una nueva clase de partícula. Probablemente se debía al hecho de que el número de partículas elementales conocidas se limitaba a tres (protones, electrones y fotones) cuyas interacciones todavía eran mal conocidas. La complicación de la estructura atómica parecía, pues, intolerable. En pocos años, la situación cambiaría completamente.

\section{LA EXPLICAGión DE LA DESINTEgRAGión $\beta$}

La desintegración $\beta$ no podía comprenderse adecuadamente hasta que no se encontraran algunas ideas clave acerca de la estructura del núcleo atómico. Retrospectivamente podemos constatar que uno de los mayores obstáculos era la hipótesis de que los electrones formaban parte del núcleo. Bohr fue uno de los primeros en darse cuenta de que había que desechar esa hipótesis. En octubre de 1931, en un congreso de físi-

8 Una de las razones de ello era que no se había determinado que los núcleos atómicos no contienen electrones. Cuando Chadwick publicó su interpretación sobre el descubrimiento del neutrón en 1932, supuso que era una partícula compuesta por un protón y un electrón (cf. Chadwick 1932b). 
ca nuclear realizado en Roma, después de examinar los problemas sin solución sobre la radiactividad, sostuvo que la situación era tal que "nos vemos conducidos a considerar la captura o la expulsión de un electrón por un núcleo, respectivamente, como una aniquilación o una creación del electrón en tanto entidad cuántica" (Bohr, 1932, p. 128). Inmediatamente después de esa afirmación sugería que esta era una buena razón para pensar que dichos procesos nucleares no obedecían a las leyes de conservación de la energía y el momento. La idea de la creación de electrones ya se encontraba en Bohr (cf. 1986 [1929], p. 88).

En 1932, James Chadwick (1932a, 1932b) descubrió una nueva clase de partícula masiva sin carga eléctrica y la bautizó con el mismo nombre de neutrón que había usado Pauli. La concibió, siguiendo una antigua conjetura de Rutherford (1920), como compuesta por un electrón y un protón. ${ }^{9}$ Durante un tiempo, hubo cierta confusión entre las partículas de Pauli y Chadwick. Sin embargo, parece claro que no podían ser la misma clase de partícula. Ambas eran neutras, pero el neutrón de Chadwick tenía una masa ligeramente superior a la del protón, mientras que la partícula de Pauli debía ser de masa nula o bien muy pequeña, no mayor que la del electrón. Si el neutrino tuviera una masa como la del neutrón, interactuaría con otros átomos de materia al ser emitido en la desintegración $\beta$. En ese caso, ocasionaría un proceso de ionización y dejaría rastros en una cámara de niebla, con lo que podría observarse, tal como se observan los protones y electrones.

Parecía evidente que el neutrón debía considerarse como parte del núcleo atómico, pero no era en absoluto claro si se trataba de una partícula elemental o no. En el mismo año 1932, Werner Heisenberg elaboró una compleja teoría del núcleo atómico, donde lo consideraba compuesto por protones y neutrones. Supuso, además, que el protón era una partícula elemental, pero el neutrón una partícula compuesta por un protón y un electrón. También sugirió que la desintegración $\beta$ podía explicarse suponiendo que el neutrón se desintegraba espontáneamente en un protón y un electrón (cf. Heisenberg, 1932a, 1932b, 1932c). En julio de 1933, Pauli, en una carta a Heisenberg, afirmó que el neutrón no podía desintegrarse de esta manera, sino que debía producir un protón, un electrón y un neutrino (cf. Pais, 1988, p. 413-7).

Simultáneamente, Dmitri Iwanenko sostuvo que el neutrón debía concebirse como una partícula elemental de spin $1 / 2$ y que, de esa manera, se explicaba la anomalía de la estadística cuántica del ${ }_{7} \mathrm{~N}^{14}$, ya que el número total de fermiones del núcleo del nitrógeno sería de 14, un número par, y no de 21, como implicaba el modelo protón-

9 En ese momento, sólo se conocían la masa y la carga del neutrón, pero no el spin. Era, entonces, posible que una partícula neutra cuya masa era ligeramente superior a la del protón fuera compuesta por un protón de carga positiva y un electrón de carga negativa. Cuando se consiguió determinar que el neutrón tenía spin 1/2 (recién en 1937), la hipótesis del compuesto protón-electrón quedó descartada. 
electrón (cf. Iwanenko, 1932, p. 4411). ${ }^{10}$ Respecto de los electrones emitidos en la desintegración $\beta$, conjeturó que todos los electrones intranucleares podían concebirse de manera análoga a los fotones absorbidos por la materia. De esa manera, era posible pensar que "la expulsión de un electrón $\beta$ era similar al nacimiento de una nueva partícula que, en estado de absorción, no posee individualidad" (Iwanenko, 1932, p. 440).

En 1933, Enrico Fermi, después de presenciar las discusiones entre Pauli y Heisenberg en el Séptimo Congreso Solvay de octubre de ese año, consiguió poner juntas muchas de las piezas que otros habían provisto y resolver el rompecabezas de la desintegración $\beta$. Su primer trabajo sobre el tema se publicó en italiano (cf. Fermi, 1933), después de que una versión inglesa fuera rechazada por Nature, que la consideró demasiado especulativa. Al comienzo de ese trabajo, se bautiza como "neutrino" a la partícula que Pauli había llamado neutrón. Es interesante citar in extenso el correspondiente pasaje:

Es bien sabido que al intentar construir una teoría de los rayos $\beta$ se encuentra una primera dificultad que depende del hecho de que los rayos Beta salen de los núcleos radioactivos con una distribución continua de velocidad que se extiende hasta una cierta velocidad máxima: lo que a primera vista no parece conciliable con el principio de la conservación de la energía. Una posibilidad cualitativa de explicar los hechos, sin tener que abandonar el principio de la conservación de la energía, consiste, según Pauli, en admitir la existencia del llamado "neutrino", esto es, de un corpúsculo eléctricamente neutro con masa del orden de magnitud de la del electrón o menor. En toda desintegración $\beta$, se tendría emisión simultánea de un electrón y un neutrino; y la energía liberada en el proceso se repartiría entre los dos corpúsculos precisamente de modo tal que la energía del electrón pueda tomar todos los valores desde o hasta un cierto máximo. El neutrino, por otra parte, a causa de su neutralidad eléctrica y de la pequeñísima masa, tendría un poder penetrante tan elevado como para escapar prácticamente de todo método actual de observación (Fermi 1933, p. 491).

Una buena parte del éxito de la teoría de Fermi se debió al hecho de que corrigió varias hipótesis erróneas acerca de la estructura del núcleo atómico y de las partículas elementales. Desde 1930, existía un estado de confusión acerca de la estructura del núcleo y puede decirse que el modelo protón-electrón se encontraba en crisis, ya que tenía muchas otras anomalías, además de las propias de la desintegración $\beta$. Por ejem-

10 Chadwick (1932b) ya lo había advertido por su cuenta, pero rechazó la idea de que el neutrón fuera una partícula elemental. 
plo tenía dificultades para explicar por qué los electrones intranucleares permanecen ligados al núcleo Fermi (1932) tenía clara conciencia de esos problemas, pero no disponia aún de un modelo alternativo.

Pauli había explicado todas las anomalías de la desintegración $\beta$, pero al costo de formular un modelo del núcleo atómico (y del proceso de desintegración mismo) que resultó imposible de mantener más allá de 1932. Después del descubrimiento del neutrón, era evidente que el modelo de Pauli (que podría llamarse protón-electrón-neutrino) tenía que abandonarse o modificarse drásticamente, ya que no podía acomodar a esta nueva clase de partícula.

El modelo del núcleo atómico de Heisenberg (cf. 1932a, 1932b, 1932c) (como el de Iwanenko), por su parte, consigue explicar la anomalía del cambio de estadística sin emplear la hipótesis del neutrino. De acuerdo con ese modelo, el núcleo de un átomo contiene un número de $Z$ protones y $A-Z$ neutrones, pero no contiene electrones. Por tanto, el núcleo del ${ }_{7} \mathrm{~N}^{14}$ está formado por 7 protones y 7 neutrones, lo cual da un número par de 14 fermiones, esto es spin entero y, consiguientemente, se le aplica la estadística de Bose-Einstein, en coincidencia con las observaciones del espectro. No obstante, sin recurrir al neutrino, no puede explicar la aparente violación de las leyes de conservación de la energía y del spin, ni tampoco aclarar el mecanismo específico de la desintegración $\beta$.

Si se emplean a la vez el modelo de Heisenberg (o el de Iwanenko) del núcleo atómico y la hipótesis del neutrino, tal como la concebía Pauli, se produce nuevamente la anomalía del cambio en la estadística cuántica. Mientras se acepte que el neutrino preexiste en el núcleo, éste vuelve a tener un número impar de fermiones y un spin fraccionario. Sólo Fermi consiguió resolver el enigma en 1933, conciliando el modelo protón-neutrón de Heisenberg e Iwanenko con la hipótesis de Pauli.

Hacia 1933, la mayoría de los físicos todavía creía que los electrones formaban parte del núcleo y que el neutrón era una partícula compuesta por un electrón y un protón. Fermi, por el contrario, adoptó la hipótesis de Heisenberg, según la cual el núcleo está formado solamente por protones y neutrones, y supuso, además, como había hecho Iwanenko y contrariamente a Heisenberg, que el neutrón no era una partícula compuesta, sino elemental dotada de spin $1 / 2$. De esa manera, quedaba resuelta la anomalía del cambio de estadística. ${ }^{11}$ Además, puesto que no había electrón alguno en el núcleo, los rayos $\beta$ debían crearse en el proceso mismo de desintegración. Fermi

11 De hecho, Fermi no la tenía en cuenta en sus artículos de 1933 y 1934. Las anomalías que mencionaba eran las del espectro continuo y la del confinamiento de los electrones en el núcleo (Fermi, 1934a, p. 1). El modelo protónneutrón del núcleo lo tomó directamente de los trabajos de Heisenberg de 1932. No cita, en cambio, el artículo de Iwanenko (1932) que, aparentemente, no conocía. La hipótesis de que el neutrón es una partícula elemental la formuló de manera independiente. 
conjeturó que procedían de la desintegración del neutrón, que daba como resultado un protón, un electrón y un neutrino $(n \rightarrow p+e+v)$. También sostuvo que los neutrinos no preexistían en el núcleo, como había pensado Pauli, sino que se creaban durante la desintegración $\beta$. Todas esas hipótesis resultaron esencialmente correctas, como es fácil apreciarlo de manera retrospectiva. Sin embargo, en 1933 las hipótesis de Fermi eran auténticas conjeturas audaces para las cuales no se disponía de mucho apoyo empírico, como él mismo reconocía.

La hipótesis de que los electrones no existen en el núcleo fue la clave que permitió la explicación de la desintegración $\beta$. Fermi tuvo en claro desde el comienzo que esa era su idea fundamental y la expresó de esta manera en el resumen de su primer artículo sobre el tema:

(...) teoría de la emisión de los rayos Beta de las sustancias radiactivas, fundada sobre la hipótesis de que los electrones emitidos por los núcleos no existen antes de la desintegración sino que vienen formados, junto con un neutrino, de un modo análogo a la formación de un cuanto de luz que acompaña un salto cuántico de un átomo (Fermi, 1933, p. 491).

Evidentemente, como allí mismo se expresa, Fermi concibió esta hipótesis por analogía con la electrodinámica cuántica, donde los electrones pueden emitir o absorber fotones. En uno de sus artículos de 1934, desarrolló la analogía de manera más explícita y señaló la consecuencia de que el número de neutrinos y electrones no se conservaba.

Para comprender la posibilidad de la emisión de los rayos $\beta$, intentaremos construir una teoría de la emisión de las partículas ligeras de un núcleo en analogía con la teoría de la emisión de un cuanto de luz de un átomo excitado en el proceso ordinario de la radiación. En la teoría de la radiación el número total de cuantos de luz no es constante; los cuantos vienen creados en el acto de su emisión de un átomo excitado, y desaparecen cuando son absorbidos. En analogía con esto, buscaremos fundar la teoría de los rayos $\beta$ sobre las siguientes hipótesis: (a) el número total de electrones y neutrinos no es necesariamente constante. Los electrones (o neutrinos) pueden ser creados o destruidos (Fermi, 1934a, p. 1-2).

Pocas veces se encuentra un relato tan explícito de los orígenes de un descubrimiento. Fermi no se limitó a emplear la analogía, sino que, además, tuvo la feliz idea de aplicar la teoría cuántica de campos, que, en ese momento, tenía un desarrollo apenas incipiente, para explicar el proceso de desintegración $\beta$. Al hacerlo, fue el primero en postular la existencia de un campo cuantizado de $\operatorname{spin} 1 / 2$, adelantándose unos meses a 
Heisenberg, que trabajaba con las mismas herramientas conceptuales en la explicación del recientemente descubierto positrón. El resultado fue una teoría de la desintegración $\beta$ que, con algunas modificaciones, tuvo vigencia por varias décadas, y que impulsó el estudio de las interacciones nucleares, todavía desconocidas. ${ }^{\mathbf{2}}$

La teoría de Fermi predecía acertadamente, dentro del margen de error observacional, todos los datos experimentales disponibles acerca de la desintegración $\beta$. Por ejemplo, permitía explicar no sólo el carácter continuo, sino también la forma del espectro observado de la energía de los electrones.

Sin embargo, nadie sabía en ese momento cómo podrían observarse los neutrinos. No se podía concebir ningún instrumento capaz de detectar la presencia de neutrinos libres, es decir, tal como emergen de los núcleos radiactivos. Dadas las propiedades del neutrino (carga eléctrica y masa nulas), éste prácticamente no interactúa con la materia. Ningún detector de partículas podría capturarlos, ya que los neutrinos lo atravesarían como si fuera espacio vacío. No obstante, en razón del carácter probabilístico de los procesos subatómicos, y cuánticos en general, la probabilidad de interacción entre neutrinos y núcleos atómicos de materia ordinaria, aunque extremadamente baja, no es nula. En 1934, inmediatamente después de que Fermi propusiera su teoría, Hans Bethe y Rudolf Peierls calcularon la probabilidad de que el neutrino interaccionara con un blanco material en un detector. La sección eficaz del neutrino, que es una medida de esa probabilidad, resultó del orden de $1{ }^{10}-{ }^{44} \mathrm{~cm}^{2}$, un valor tan bajo como jamás se había encontrado en el dominio de la microfísica. Ante ese resultado, Bethe y Peierls concluyeron que "si el neutrino no tiene interacción con otras partículas (...), no hay ningún modo prácticamente posible de observar el neutrino" (Bethe \& Peierls, 1934, p. 532).

Dos años después, en 1936, el mismo Bethe junto con Robert Bacher hicieron una revisión detallada del estado de la física de partículas en ese momento y comentaron la posible observación experimental del neutrino en los siguientes términos:

(...) parece físicamente imposible detectar neutrinos en estado libre, esto es, después de que han sido emitidos por un átomo radiactivo. Hay un solo proceso que los neutrinos ciertamente pueden causar. Es el proceso $\beta$ inverso, que consiste en la captura de un neutrino por un núcleo junto con la emisión de un electrón (o positrón). Este proceso es tan extremadamente raro que un neutrino tiene que atravesar, en promedio, $10^{16} \mathrm{~km}$ de materia sólida antes de causar tal proceso.

12 Sobre el proceso de desarrollo de las teorías de las fuerzas nucleares fuerte y débil, véase Brown \& Rechenberg (1996), que ofrece un panorama muy completo. La interacción nuclear débil es la responsable de la desintegración $\beta$. Fermi, pionero en el descubrimiento de esa fuerza, no volvió a escribir sobre la desintegración $\beta$ después de 1934, cuando publicó versiones más completas de su teoría (cf. Fermi, 1934a, 1934b). 
Los métodos actuales de detección deben mejorarse en sensibilidad al menos por un factor de $10^{13}$ antes de que tal proceso pueda detectarse (Bethe \& Bacher, 1936, p. 188).

La desintegración $\beta$ inversa se concebía como el proceso en el cual un neutrino interacciona con un protón y produce un neutrón y un positrón $(v+p \rightarrow n+e+)$. Ni el antineutrino incidente ni el positrón resultante se pueden observar en un detector de partículas. El neutrino, porque no deja huella alguna antes de interaccionar con el protón, y el positrón, porque se aniquila inmediatamente al encontrarse con un electrón de cualquier átomo de materia. La aniquilación del par electrón-positrón produce dos rayos $\gamma$ (fotones de alta energía) que se mueven en direcciones opuestas. Estos rayos $\gamma$ emergen en un tiempo característico calculable y con una energía determinada también predecible, por lo cual, en principio, son observables mediante detectores de luz colocados a cada lado del aparato en que se produce la interacción entre neutrinos y protones. Este era un experimento posible para detectar el neutrino, pero la tecnología para su realización efectiva sólo estuvo disponible casi dos décadas después del cálculo de Bethe y Bacher.

Hacia 1936, el debate acerca de la conservación de la energía en los procesos subatómicos, y en la desintegración $\beta$ en particular, seguía inconcluso tanto desde el punto de vista teórico como experimental. Un experimento realizado ese año por Robert Shankland (1936) parecía mostrar que el momento y la energía no se conservaban en la interacción de los fotones con los electrones. La interpretación de ese resultado experimental generó una viva discusión entre los físicos teóricos. Bohr y Dirac adoptaron posiciones opuestas, que representan una inversión de sus opiniones anteriores. Dirac (1936), sorprendentemente, se declaró dispuesto a admitir que había que renunciar a las leyes de conservación con el fin de construir una electrodinámica cuántica satisfactoria. A la vez, se mostró escéptico respecto de la supuesta existencia del neutrino. Bohr, en cambio, renunció a cuestionar las leyes de conservación, como lo había hecho hasta entonces, y apoyó enérgicamente la teoría de Fermi. Terminaba su breve artículo de 1936 de esta manera:

finalmente, debe señalarse que los fundamentos de las serias dudas acerca de la estricta validez de las leyes de conservación en el problema de la emisión de los rayos $\beta$ desde los núcleos atómicos se encuentran ahora ampliamente eliminados, por el sugestivo acuerdo entre la evidencia experimental rápidamente creciente acerca de los fenómenos de radiación $\beta$ y las consecuencias de la hipótesis del neutrino de Pauli tan admirablemente desarrolladas en la teoría de Fermi (Bohr, 1936, p. 26). 
La afirmación de Bohr era bastante temeraria en ese momento puesto que la existencia del neutrino no se había comprobado y, además, no había en la práctica ninguna posibilidad de realizar un experimento que permitiera detectarla. ${ }^{\mathbf{1 3}}$

El éxito explicativo de la teoría de Fermi fue tal que convenció a la gran mayoría de los físicos de que la hipótesis del neutrino debía considerarse confirmada. Hacia comienzos de la década de 1950, la teoría de Fermi se había desarrollado hasta volverse una parte aceptada de la física de partículas. La teoría explicaba satisfactoriamente numerosos fenómenos del ámbito de la desintegración $\beta$, como la forma exacta del espectro de energía de los electrones, y había sido confirmada por múltiples experimentos de laboratorio. Estos hechos convencieron a la mayoría de los físicos de partículas acerca de la existencia real del neutrino De acuerdo con múltiples testimonios, la hipótesis del neutrino tenía amplia aceptación en la comunidad de los físicos antes de que esta partícula se observara por primera vez (cf. Allen, 1958, p. v; Pais, 1988, p. 569; Reines, 1995, p. 203; Arns, 2001, p. 329-31; Franklin, 2004, p. 159). ${ }^{14}$ Sin embargo, el hecho mismo de que en ese momento se proyectaran experimentos para detectar neutrinos libres indica que todavía quedaban dudas, justificadamente en mi opinión, acerca de su existencia.

Cowan y Reines diseñaron, en 1953, un experimento destinado a la observación de neutrinos libres, aprovechando la nueva tecnología disponible en los reactores nucleares de fisión. Estos reactores eran capaces de producir una corriente de millones de neutrinos que podía controlarse a voluntad. La probabilidad de interacción de un único neutrino incidente en un blanco material es, como ya indicaban los cálculos de Bethe y Peierls, extremadamente baja. Pero la probabilidad de que se produzca alguna interacción cuando inciden millones de neutrinos por segundo sobre una gran cantidad de materia (por ejemplo, un tanque de agua) es razonablemente alta.

En el brevísimo artículo de febrero de 1953, en el que anunciaban la realización de su experimento, Reines y Cowan decían lo siguiente:

el éxito de la hipótesis del neutrino en la explicación de los hechos observados de la desintegración Beta provee una evidencia razonablemente convincente de la existencia del neutrino. Sin embargo, la observación de un efecto producido por un neutrino en una localización diferente de su lugar de origen sería interesante porque (1) toda duda respecto de su existencia sería resuelta, y (2) podría obtenerse más información acerca de sus propiedades y su lugar en la naturaleza de las cosas (Reines \& Cowan, 1953a, p. 492).

13 La idea original de Bohr de que la energía sólo se conserva estadísticamente no se extinguió del todo. Schrödinger (1958) todavía la consideraba posible.

14. Sería importante corroborar esta aceptación mediante un examen sistemático de los libros de texto de la física de partículas de la década de 194ㅇ, pero esa tarea está más allá de los límites de este trabajo. 
Esta afirmación indica dos cosas importantes. En primer lugar, ambos físicos creían que el éxito explicativo de la teoría de Fermi proporcionaba buenas razones para creer en la hipótesis del neutrino, pero que no era suficiente para afirmar categóricamente la existencia de esa clase de partículas. En segundo lugar, consideraban que la observación de un neutrino libre, interactuando con un detector colocado a distancia del núcleo que emitía el neutrino, sería efectivamente una evidencia suficiente de su existencia, al menos, más allá de toda duda razonable.

\section{Reflexiones ePistemológicas}

Disponemos ahora de la información básica como para hacer una evaluación epistemológica de las dos primeras etapas del proceso que culminó en el descubrimiento del neutrino. Intentaré, entonces, responder a las tres preguntas formuladas al comienzo.

La primera pregunta se refiere a la etapa de invención de la hipótesis. ¿Qué tipo de argumentos se utilizaron para justificar la postulación de la hipótesis del neutrino? Para obtener una respuesta satisfactoria es necesario aclarar previamente el carácter de la propia hipótesis del neutrino. Ante todo, si entendemos por hipótesis ad hoc aquellas que se proponen con el fin explícito de evitar la refutación de una teoría o hipótesis previamente aceptada, entonces, la hipótesis del neutrino se originó de manera claramente $a d$ hoc. Pauli quiso mantener las leyes de conservación de la energía y el momento angular, ampliamente confirmadas por los más diversos fenómenos físicos, frente a la aparente refutación que proporcionaba la desintegración $\beta$. Las propiedades del neutrino, como la carga eléctrica nula y el $\operatorname{spin} 1 / 2$, se eligieron específicamente para acomodar los hechos observados preservando las leyes de conservación. Si, en cambio, entendemos por hipótesis $a d$ hoc aquellas que no son contrastables ni agregan contenido empírico a un sistema de hipótesis, entonces, la hipótesis de Pauli no era en absoluto ad hoc. La hipótesis del neutrino tiene consecuencias observacionales novedosas, al menos en el contexto de una teoría como la de Fermi.

La interpretación más corriente de la invención de la hipótesis del neutrino la considera un ejemplo de abducción o inferencia a la mejor explicación. El episodio, en efecto, encaja bien en el esquema de Peirce y Hanson de una inferencia abductiva o retroducción. ${ }^{15}$ En primer lugar, se observa un fenómeno sorprendente, a saber, el espectro continuo de la desintegración $\beta$, que parece violar leyes de conservación bien

15 Peirce (1903) y Hanson (1958), que se inspira directamente en Peirce. Hanson considera a la hipótesis del neutrino como un caso paradigmático de retroducción (cf. Hanson, 1958, p. 124-5). Aquí no distinguiré entre abducción e inferencia a la mejor explicación (cf. Lipton ,2004). 
establecidas. Después, se inventa una hipótesis tal que, si fuera verdadera, el fenómeno en cuestión sería comprensible y perdería su carácter anómalo: en la desintegración $\beta$, se crea una partícula inobservada, el neutrino, que acarrea la energía y el momento faltantes en los productos finales de la reacción. Por último, se postula la hipótesis en cuestión: existen los neutrinos, que tienen como propiedades características el spin $1 / 2$, la carga eléctrica nula y la masa en reposo nula o menor que la el electrón. Esta es una reconstrucción perfectamente posible del proceso de invención de la hipótesis del neutrino y, en tanto reconstrucción, me parece inobjetable.

Si nos atenemos al relato del propio Pauli, en cambio, obtenemos una descripción bastante diferente. Como señalamos antes, la invención del neutrino se basó en un argumento analógico con la conservación de la carga eléctrica. Se podría haber utilizado un argumento similar para apoyar la conservación de la paridad, que, como se sabe, se demostró experimentalmente como una cantidad no conservada, poco después de los experimentos de Cowan y Reines (cf. Wu et al., 1957). Pero el argumento, aunque falible, presta indudable apoyo inductivo a la conclusión. Puesto que las leyes de conservación de la carga, la energía y el momento, que son independientes entre sí, se habían encontrado asociadas en numerosos fenómenos físicos, era plausible, aunque no necesario, suponer que también estarían asociadas en el fenómeno de la desintegración $\beta$. Esto responde a la primera pregunta, hasta donde es posible indagar el proceso de invención de hipótesis nuevas. Sobre la manera en que a Pauli se le ocurrió la hipótesis del neutrino, nada puede decirse desde el punto de vista epistemológico. El propio Pauli guarda silencio al respecto.

La segunda pregunta también corresponde a la etapa que he llamado de invención de la hipótesis del neutrino. ¿Cómo debe evaluarse epistemológicamente esta hipótesis? La pregunta no se refiere, sin embargo, al proceso de generación de dicha hipótesis, sino al ámbito que Laudan ha llamado "contexto de prosecución" de las teorías (cf. Laudan, 1977, p. 109-14). Dicho contexto se relaciona con el cálculo de los costos y beneficios de adoptar una hipótesis nueva como herramienta de trabajo, desarrollarla o incorporarla a una teoría o sistema de hipótesis y extraer sus consecuencias lógicas. Esta evaluación casi siempre es comparativa, es decir, relativa a las hipótesis o teorías rivales disponibles en un momento dado. En el caso del neutrino, el juicio de Pauli es claramente comparativo. Había una sola hipótesis rival, la de Bohr, que postulaba la violación de las leyes de conservación de la energía y el momento en los procesos subatómicos. Los costos y beneficios de cada una de estas hipótesis se pueden evaluar en términos de revisión de las teorías existentes y simplicidad global del conocimiento físico, así como de otras virtudes epistémicas. De esa manera, la aceptación de una teoría es más costosa si implica la revisión de otras teorías o hipótesis aceptadas y, además, complica el conocimiento, por ejemplo, aumentando el número 
de teorías independientes que no se pueden unificar. Como ya hemos señalado, la hipótesis de Pauli resulta favorecida en ambos aspectos. Si el conservadurismo se considera una virtud epistemológica, como hacen Quine y otros filósofos (cf. Quine \& Ullian, 1978, p. 66-8), la hipótesis de Pauli tiene la virtud adicional de ser la más conservadora, por audaz que haya parecido en su tiempo.

La tercera pregunta concierne a la relación entre las virtudes explicativas de la hipótesis del neutrino y la aceptación de la existencia de neutrinos. Es indudable que la hipótesis del neutrino, en el contexto de la teoría de Fermi, proporcionó una explicación satisfactoria de diversos aspectos del fenómeno de la desintegración $\beta$. A su vez, la teoría de Fermi resultó confirmada por diversos fenómenos nuevos descubiertos en el campo de la radiactividad. Allan Franklin considera que estos fenómenos también confirman a la hipótesis del neutrino por sí misma. Dice al respecto:

la teoría de Fermi de la desintegración $\beta$, que incorporaba al neutrino de una manera esencial, tenía un apoyo evidencial muy fuerte. Ese apoyo también proporcionaba buenas razones para creer en la realidad del neutrino. El argumento filosófico es el siguiente. Si varios enunciados, o supuestos, tomados conjuntamente implican un resultado experimental, entonces, la observación de ese resultado apoya a la conjunción de esos enunciados así como a cada uno de ellos separadamente. En el caso de la teoría de Fermi, esto incluye la existencia del neutrino (Franklin, 2004, p. 180).

Este argumento no es concluyente en razón del holismo epistemológico, que Franklin pasa por alto. Cuando un sistema de hipótesis implica una consecuencia observacional (que no es implicada por ningún subconjunto propio de hipótesis de ese sistema), la verificación de dicha consecuencia confirma a todo el sistema de manera global. La confirmación no puede distribuirse a cada una de las hipótesis componentes. Por esta razón, los fenómenos de la desintegración $\beta$ no confirman a la hipótesis del neutrino de manera independiente del resto de la teoría de Fermi, la cual incluye muchas otras hipótesis, por ejemplo, acerca de la estructura del núcleo atómico.

El éxito explicativo de la teoría de Fermi, según creo, no era suficiente para aceptar la existencia del neutrino. La comunidad de los físicos se apresuró en aceptarla y, al hacerlo, no procedió de manera completamente razonable. ${ }^{\mathbf{1 6}}$ La lección del planeta Vulcano debería haberse aprendido de una vez y para siempre. Como se sabe, en 1859 , después de haber descubierto la célebre anomalía en el avance del perihelio de Mercu-

16 Es justo decir que no todos los físicos aceptaban la existencia del neutrino, aunque los escépticos eran minoría hacia $195^{\circ}$. 
rio, Jean Leverrier postuló la existencia del planeta Vulcano, que orbitaría entre Mercurio y el Sol, y sería el causante de las perturbaciones observadas. Procedía tal como lo había hecho en 1846, cuando postuló la existencia de Neptuno para explicar anomalías en la órbita de Urano. El planeta Neptuno se descubrió inmediatamente, pero las observaciones telescópicas, después de medio siglo de búsqueda infructuosa, nunca permitieron encontrar el menor indicio de la existencia de Vulcano. Hubiera sido irracional aceptar la existencia de Neptuno por el sólo hecho de que proporcionaba una explicación (aunque fuera la mejor explicación disponible) de las anomalías observadas en la órbita de Urano. La existencia de Neptuno sólo podía resultar aceptable, como ocurrió de hecho, después de haberlo observado. Y, por cierto, nadie aceptó la existencia de Vulcano. Aunque esta última hipótesis no tenía rivales y era explicativamente satisfactoria (al menos tanto como la de Neptuno), nadie consideró que ese éxito explicativo fuera suficiente como para aceptar que Vulcano era real. ${ }^{\mathbf{1 7}}$

En general, el éxito explicativo de una hipótesis no constituye por sí solo eviden-cia suficiente de que las entidades postuladas por dichas hipótesis realmente existen. No importa cuán bien confirmada esté la teoría que contiene a esas hipótesis, o, incluso, si algunas de las entidades postuladas por la teoría se descubren por observación. Esto es, si la teoría $T$ predice la existencia de ciertos tipos de entidades, $X, Y, Z$, hasta el momento desconocidas, el descubrimiento de $X$ e $Y$ no constituye evidencia suficiente para aceptar la existencia de $Z$, aunque, por supuesto, tal descubrimiento confirma globalmente a la teoría $T$. Para afirmar la existencia de una clase de entidades desconocida se requiere evidencia observacional de que dichas entidades existen. Típicamente, se requiere la observación de algún efecto asociado unívocamente a dicha clase de entidades.

La teoría electrodébil constituye un buen ejemplo de lo que acabo de afirmar. Entre 1967 y 1968, Abdus Salam y Steven Weinberg elaboraron una teoría unificada de las interacciones electromagnética y nuclear débil. ${ }^{\mathbf{8}}$ La síntesis electrodébil, como se la llama actualmente, predecía la existencia de nuevos tipos de partículas: los bosones vectoriales $W+, W$ " y $Z$ o, mediadores de la interacción débil, y el bosón de Higgs, que por medio de un complejo mecanismo, proporciona las masas a estasúltimas. Los bosones vectoriales $W+, W$ "y Zo se descubrieron experimentalmente en 1983, debido al trabajo de un enorme equipo de científicos y técnicos dirigido por Carlo Rubbia (cf.

17 De hecho, la anomalía en el avance del perihelio de Mercurio permaneció sin explicar hasta 1915, cuando la teoría de la relatividad general de Einstein dio la explicación actualmente aceptada. Roseveare (1982) es una historia detallada de las diferentes hipótesis que se propusieron para explicar esta anomalía de la mecánica celeste newtoniana. 18 Ésta teoría, dicho sea de paso, reemplazó a la teoría de Fermi de la interacción débil. Para una exposición introductoria de la teoría electrodébil, véase Coughlan \& Dodd, (2006, p. 99-12). En las páginas 71-98 de la misma obra se encuentra también una exposición de la teoría de Fermi y de sus desarrollos posteriores. Sobre la histora de la síntesis electrodébil, véase Hoddeson et al., (1997), donde hay amplias referencias a las fuentes. 
Arnison et al., 1983). ${ }^{19}$ El bosón de Higgs, una partícula de spin o y masa desconocida, aunque presumiblemente muy grande, no se observó hasta el momento, a pesar de los numerosos intentos realizados en diversas partes del mundo. ${ }^{20}$ La teoría electrodébil se encuentra muy bien confirmada en la actualidad, probablemente más que la teoría de Fermi a comienzos de la década de 1950. La existencia de los bosones vectoriales $W+, W$ " yZo se acepta desde 1983 y este hecho se encuentra incorporado como parte del conocimiento vigente en todos los libros de texto de física de partículas. Ningún libro de texto, sin embargo, afirma de manera categórica la existencia del bosón de Higgs, por más que su existencia es tan indispensable para la física de partículas actual como lo era la del neutrino después de la teoría de Fermi. Por el contrario, el descubrimiento de esta partícula se considera una de las tareas fundamentales de la física experimental de nuestros días (cf. Martin, 2009, p. 187-8, 3०5-11; Das \& Ferbel, 2003, p. 353-5).

La actitud más razonable de los físicos antes de los experimentos de Cowan y Reines, iniciados en 1953, era la suspensión del juicio acerca de la existencia del neutrino hasta que se dispusiera de suficiente evidencia observacional. Esa misma actitud tienen actualmente los físicos de partículas respecto del bosón de Higgs y de muchas otras partículas postuladas por teorías más tentativas y menos confirmadas que la síntesis electrodébil. ${ }^{21}$ No se sigue de ello que deban abandonarse tales hipótesis, ni mucho menos las teorías a las cuales pertenecen. Por el contrario, cuando una teoría resulta explicativamente exitosa, el curso de acción más razonable consiste en emprender experimentos que puedan eventualmente confirmarla. Así, dado el éxito explicativo de la hipótesis del neutrino, los experimentos de Cowan y Reines estaban plenamente justificados. Sólo las limitaciones de la tecnología de la época impidieron que éstos se efectuaran mucho antes.

El primer experimento dirigido a detectar neutrinos libres, realizado en 1953, tuvo resultados inconcluyentes (cf. Reines \& Cowan, 1953b). En 1956, Cowan y Reines realizaron un segundo experimento mucho más exitoso y anunciaron formalmente que el neutrino había sido observado (cf. Cowan et al., 1956; Reines \& Cowan, 1956). Los resultados completos del análisis de sus datos, sin embargo, no se publicaron hasta 1960 (Reines et. al., 1960). Sólo en ese momento, en mi opinión, la comunidad científica dispuso de una evidencia suficiente como para afirmar la existencia del neutrino.

19 Después de esa fecha, se encontraron nuevas evidencias en otros experimentos (cf. Watkins, 1986).

20 De hecho, dado el tamaño, la complejidad y el costo de los instrumentos necesarios, sólo existen dos lugares donde en principio sería posible observar el bosón de Higgs: el Fermilab en los Estados Unidos de América, y el GERN, situado en Suiza, pero construido por toda la Comunidad Económica Europea. El estado actual de la búsqueda de esta partícula puede seguirse en las respectivas páginas Web del Fermilab y del CERN.

21 Entre otras muchas partículas hipotéticas, podemos mencionar a los monopolos magnéticos, cuya existencia predicen las teorías unificadas de la interacción electrodébil y la interacción nuclear fuerte, y a los gravitones, postulados por diversas teorías cuánticas de la gravitación. 


\section{Conclusión}

A partir del análisis epistemológico que hemos realizado en el parágrafo anterior, podemos resumir nuestras conclusiones de la siguiente manera.

La hipótesis del neutrino de Pauli era la más conservadora cuando éste la propuso a fines de 1930. La única hipótesis alternativa en ese momento, la de Bohr, que proponía la no conservación de la energía y el momento angular en los procesos microscópicos individuales, era mucho más radical e implicaba revisiones mucho más drásticas de las teorías físicas entonces vigentes. Otras hipótesis más conservadoras que la de Pauli, como la de las interacciones con partículas fuera del núcleo radiactivo, habían sido disconfirmadas experimentalmente. La hipótesis de Pauli era compatible con la física cuántica y de partículas tal como se encontraba, en algunos casos en estado de confusión, después de 1930. El mayor problema de la hipótesis del neutrino era el supuesto de que los electrones y los neutrinos emitidos en la desintegración $\beta$ preexistían en el núcleo. Una vez que Fermi se deshizo de esta hipótesis, fue posible formular una teoría coherente de la desintegración $\beta$, que incorporaba las ideas fundamentales de Pauli acerca del neutrino, pero rechazaba el modelo del núcleo atómico en el que se había apoyado.

La hipótesis de Pauli era indudablemente ad hoc cuando la inventó. Las propiedades del neutrino las eligió precisamente para dar cuenta de todos los fenómenos observados sobre la desintegración $\beta$ y, al mismo tiempo, para salvar las leyes de conservación amenazadas y mantener las que se cumplían. El neutrino debía tener spin $1 / 2$ para salvar la ley de conservación del momento angular; un espectro de energía continuo y complementario (en el sentido ya explicado) al del electrón emitido para salvar la ley de conservación de la energía; carga nula para mantener la ley de conservación de la carga eléctrica; y masa nula o menor que la del electrón para explicar por qué no interactuaba con los átomos de materia y, por tanto, no dejaba huellas en los detectores de partículas.

El problema del cambio en la estadística cuántica, que fue uno de los principales motivos que impulsaron a Pauli, era en realidad un artefacto del modelo protón-electrón del núcleo atómico, todavía vigente en 1930. La anomalía quedó resuelta en 1932 mediante el modelo protón-neutrón de Heisenberg e Iwanenko, sin necesidad de suponer la existencia del neutrino. Pauli la resolvió mediante un modelo del núcleo que no podía acomodar el neutrón, pero en la teoría de Fermi el problema del cambio de estadística ya no se presenta.

En el contexto de la teoría de Fermi de la desintegración $\beta$, la hipótesis del neutrino dejó de ser ad hoc, ya que permitió explicar hechos nuevos para cuya explicación no había sido inventada. El principal de esos hechos, descubierto en 1932, esto es, des- 
pués de la invención de Pauli, es la forma precisa de las curvas que describen los espectros de la energía de los electrones emitidos (cf. Sargent, 1932). La teoría de Fermi predijo de manera exacta estas curvas, que no tenían explicación teórica alguna en el momento en que fueron descubiertas.

La hipótesis del neutrino no resultaba confirmada por sí misma por los hechos nuevos que la teoría de Fermi explicaba y predecía. La confirmación de la teoría de Fermi era global y no podía extenderse a sus partes componentes.

Por último, el éxito explicativo y predictivo de la teoría de Fermi no proporcionaba razones suficientes como para afirmar la existencia de neutrinos. A lo sumo, implicaba una presunción a favor de la existencia de esta clase de partículas, pero una presunción puramente hipotética que debía confirmarse por medio de experimentos. La hipótesis del neutrino había sido inventada para explicar la desintegración $\beta$ y la teoría de Fermi era, esencialmente, una teoría de la desintegración $\beta$. La clase de evidencia observacional que se necesitaba para afirmar la realidad de los neutrinos era la detección experimental de un proceso en el cual se produjera la interacción de neutrinos con un detector de partículas relativamente alejado de los átomos radiactivos que se desintegraban. Esta evidencia recién la proporcionaron los experimentos de Cowan y Reines en 1956, pero era mucho menos firme y clara que lo que habitualmente se cree. Había importantes valores discordantes en los informes que Cowan y Reines publicaron ese año (cf. Arns, 2001). No obstante, el experimento no fue repetido por ningún otro grupo de experimentadores y la hipótesis de la existencia de neutrinos libres en la naturaleza se dio por confirmada. $\$$

Alejandro Gassini Profesor Adjunto Regular del Departamento de Filosofia, Universidad de Buenos Aires, Investigador Independiente del Consejo Nacional de Investigaciones Científicas y Técnicas, Argentina. alepafrac@yahoo.com.ar 


\begin{abstract}
By 1930 the explanation of Beta radioactive decay produced a crisis in the domain of particle physics. Conservation of energy, momentum and quantum statistics seemed to be challenged. Pauli succeeded in solving all these anomalies by postulating the existence of neutrinos inside atomic nuclei. But he did it at the cost of assuming a model of the nucleus that was untenable after 1932, when the neutron was discovered. In 1933 Fermi put forward what we still regard as the correct explanation of Beta decay. In this article I review the explanatory virtues of the neutrino hypothesis. I contend that Pauli's invention was ad hoc in 1930 but it became a testable hypothesis in the context of Fermi's theory. I then conclude by asserting that there was not enough evidence to accept the existence of the neutrino prior to Cowan and Reines' second experiment in $195^{6}$.
\end{abstract}

KEYwords • Beta decay. Neutrino. Conservation laws. Quantum statistics. Scientific discovery.

\title{
REFERENGIAS BIBLIOGRÁFIGAS
}

Allen, J. S. The neutrino. Princeton: Princeton University Press, $195^{8}$.

Arnison, G. et al. Experimental observation of isolated large transverse energy electrons with associated missing energy at $\sqrt{ }_{\mathbf{s}}=54, \mathrm{O}$ GeV. Physical Review, $122 \mathrm{~B}, \mathrm{p} .103-16,1983$.

Arns, R. G. Detecting the neutrino. Physics in Perspective, 3, p. 314-34, 2001.

Bethe, H. \& Bacher, R. Nuclear physics. Review of Modern Physics, 8, p. 82-229, 1936.

Bethe, H. \& Peierls, R. The “neutrino”. Nature, 133, p. 532, 1934.

Вонм, D. Quantum theory. New York: Dover, 1989 [1951].

Вонк, N. On the constitution of atoms and molecules. Part II. Systems. Containing a single nucleus. Philosophical Magazine, 26, p. 476-502, 1913.

. Atomic stability and conservation laws. Atti del Convegno di Fisica Nucleare della "Fondazione Alessandro Volta"-Ottobre 1931. Roma: Reale Academia d'Italia, 1932. p. 119-3o. . Conservation laws in quantum theory. Nature, 138, p. 25-6, 1936.

. B-ray spectra and energy conservation. In: PeIERLs, R. (Ed.). Collected works of Bohr, N. Nuclear physics (1929-1952). Amsterdam: North-Holland, 1986 [1929]. v. 9. p. $87^{-9}$.

. On the properties of the neutron. In: PeierLs, R. (Ed.). Collected works of Bohr, N. Nuclear physics

(1929-1952). Amsterdam: North-Holland, 1986 [1932].v. 9. p. $117-8$.

Bohr, N.; Kramers, H. \& Slater, J. The quantum theory of radiation. Philosophical Magazine, 47, p. $785^{-}$ 802,1924 .

Brown, L. \& Reghenberg, H. The origin of the concept of nuclear forces. Bristol: Institute of Physics Publishing, 1996.

Brush, S. How ideas became knowledge: the light-quantum hypothesis $1905^{-19} 35$. Historical Studies in the Physical and Biological Sciences, 37, p. 205-4,6, 2007.

Cassini, A. ¿Cómo se reconoce un descubrimiento científico? Análisis Filosófico, 21, p. 43-75, 2001.

Chadwick, J. Intensitätsverteilung im magnetischen Spektrum der $\beta$-Strahlen von Radium B+C. Verhandlungen der Deutschen Physikalischen Gesellschaft, 16, p. 383-91, 1914.

. Possible existence of a neutron. Nature, 129, p. 312, 1932a.

The existence of a neutron. Proceedings of the Royal Society of London, A. 136, p. 692-708, 1932b.

Сомpton, A. A quantum theory of the scattering of X rays by light elements. Physical Review, 21, p. 483$502,1923$. 
Coughlan, G. \& Dodd, J. The ideas of particle physics: an introduction for scientists. 3 ed. Cambridge: Cambridge University Press, 2006.

Cowan, C. et al. Detection of the free neutrino: a confirmation. Science, 124, p. 103-4, 1956.

DAs, A. \& Ferbel, T. Introduction to nuclear and particle physics. 2 ed. Singapore: World Scientific, 2003.

DirAc, P. Does conservation of energy holds in atomic processes. Nature, 137, p. 298-9, 1936.

ElLis, C. \& Wooster, W. The average energy of the disintegration of radium E. Proceedings of the Royal Society, A117, p. 109-23, 1927 .

Enz, G. P. No time to be brief: a scientific biography of Wolfgang Pauli. New York: Oxford University Press, 2002.

Fermi, E. Lo stato attuale della fisica del nucleo atomico. La Ricerca Scientifica, 3, p. 101-13, 1932. Tentativo di una teoria dell'emissione dei raggi “Beta”. La Ricerca Scientifica, 4, p. 491-5, 1933. Tentativo di una teoria dei raggi $\beta$. Il Nuovo Cimento, 11, p. 1-21, 1934a.

.Versuch einer theorie der $\beta$-Strahlen. I. Zeitschrift fûrPhysik, 88, p. 161-77, 1934,b.

Frankin, A. Selectivity and discord: two problems of experiment. Pittsburgh: University of Pittsburgh Press, 2002.

Are there really neutrinos? An evidential history. Boulder: Westview Press, 2004.

Hanson, N. R. Patterns of discovery: an inquiry into the conceptual foundation of science. Cambridge: Cambridge University Press, $195^{8}$.

Heisenberg, W. Über den Bau der Atomkerne. I. Zeitschrift für Physik, 77, p. 1-11, 1932a. Über den Bau der Atomkerne. II. Zeitschrift für Physik, 78, p. 156-64,1932b. Über den Bau der Atomkerne III. Zeitschrift für Physik, 8o, p. 587-96, 1932c.

Heitler, W. \& Herzberg, G. Gehorchen die Stickstoff-kerne der Boseschen Statistik? Naturwissenschaften, 17 , p. $673,1929$.

Hoddeson, L. et al. (Ed.). The rise of the standard model: particle Physics in the 196os and 1970s. New York: Cambridge University Press, 1997 .

Iwanenko, D. Sur la constitution des noyaux atomiques. Comptes Rendues des Séances de L'Académie des Sciences, 195 , p. 439-41, 1932 .

Jensen, C. Controversy and consensus: nuclear Beta decay 1911-1934. Basel: Birkhäuser, 2000.

KRAGH, H. The origin of radioactivity: from solvable problem to unsolved non-problem. Archive for History of Exact Sciences, 50, p. 331-58, 1997 .

LAUDAN, L. Progress and its problems: towards a theory of scientific growth. Berkeley: University of California Press, 1977 .

Lipton, P. Inference to the best explanation. 2 ed. London: Routledge, 2004.

Martin, B. R. Nuclear and particle physics. 2 ed. Chichester: John Wiley \& Sons, 2009.

PaIs, A. Inward bound: of matter and forces in the physical world. New York: Oxford University Press, 1988.

Pauli, W. The connection between spin and statistics. Physical Review, $5^{8}$ p. $7^{16-22,} 1940$.

Acerca de la anterior y la más reciente historia del neutrino. In: . Escritos sobre física y filosofía. Madrid: Debate, 1996, p. 243-74.

Peierls, R. (Ed.). Collected works of Bohr, N. Nuclearphysics (1929-1952). Amsterdam: North-Holland, 1986. v. 9.

Peirce, G. S. Pragmatism as the logic of abduction. In: Peirce Edition Projects (Ed.). The essential Peirce: selected philosophical writings. (1893-1913). Bloomington: Indiana University Press, 1903. v. 2. p. 22641.

Peirce Edition Projects (Ed.). The essential Peirce: selected philosophical writings. (1893-1913). Bloomington: Indiana University Press, 1903. V. 2.

Quine, W. V. O. \& Ullian, J. The web of belief. 2 ed. New York: Random House, 1978. 
Reines, F. The Neutrino: from poltergeist to particle. The Nobel Prizes. Stockholm: Almquist \& Wiksell, 1995. p. 202-21.

Reines, F. \& Cowan, C. A proposed experiment to detect the free neutrino. Physical Review, 90, p. 492-3, $195^{3 \mathrm{a}}$.

. Detection of the free neutrino. Physical Review, 92, p. 83o-1, $195^{3 \mathrm{~b}}$.

. The neutrino. Nature, 178 , p. 446-9, 1956.

Reines, F. et al. Detection of the free antineutrino. Physical Review, 117, p. 159-73, 1960.

Roseveare, N. T. Mercury's perihelion from Le Verrier to Einstein. Oxford: Clarendon Press, 1982.

Rutherford, E. Nuclear constitution of atoms. Proceedings of the Royal Society, A97, p. 374-4,00, 1920.

Sargent, B. Energy distribution curves of the disintegration electrons. Proceedings of the Cambridge Philosophical Society, 24, p. 538-83, 1932.

Schrödinger, E. Might perhaps energy be a statistical concept. Il Nuovo Cimento, 9, p. 162-70, $195^{8}$.

Shamos, M. H. (Ed.). Great experiments in physics: firsthand accounts from Galileo to Einstein. New York: Dover, 1987 [1959].

Shankiand, R. S. An apparent failure of the photon theory of scattering. Physical Review, 49, p. 8-13, 1936.

Solomer, N. The elusive neutrino. New York: Freeman \& Company, 1997.

Stuewer, R. The compton effect: turning point in physics. New York: Science Publications, 1975.

Watkins, P. The story of the $W$ and Z. Cambridge: Cambridge University Press, 1986.

Winter, K. (Ed.). Neutrino physics. 2 ed. Cambridge: Cambridge University Press, 2000.

Wu, C. et al. Experimental test of parity conservation in Beta decay. Physical Review, 105, p. 14.13-5, 1957.

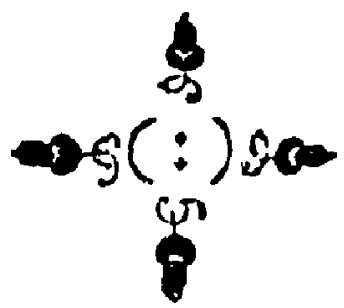

